\title{
$\mathrm{CiSj}$
}

\section{EVOLUTIONARY ENVIRONMENT FOR 3D MORPHOLOGICAL DESIGN}

\author{
A. Lamas and R.J. Duro \\ Integrated Group for Engineering \\ Universidade da Coruña \\ C/Mendizabal s/n 15403 Ferrol, (A Coruña), Spain \\ \{alamas, richard\}@udc.es \\ http://www.gii.udc.es
}

\begin{abstract}
This paper deals with the automation of morphological design. The system proposed here is a part of a complete automatic design system that considers the divergent and convergent stages of the design process through evolutionary procedures. The system provides a way to introduce aesthetics in the decision process. This is a difficult problem within an automatic design system, as aesthetics are subjective, depend on the opinions of humans and humans do not usually agree. The objective here is to be able to obtain the best possible aesthetic solutions for a given set of humans. This is achieved through the introduction of a set of man machine interfaces that allow the system to extract information on their relative opinions without explicitly asking them, and combines them with the engineering information provided by other simulators that participate in the design process.
\end{abstract}

Keywords: Automatic Design, Aesthetic Design, Evolutionary Algorithms.

\section{INTRODUCTION}

Most of the research carried out on the design process has concentrated on the optimization of certain stages through the creation of isolated computational tools that help the engineer/designer to visualize, model and/or evaluate possible solutions to a problem as a function of his/her previous experience. The lack of generality of this type of tools and the fact that they are only usable in certain stages of the design process, leaving others out, imply that they can only be classified as an aid to design and not as design systems. These tools do not contemplate two of the most important stages in design: The search for solutions (or divergent stage), which is where the solutions are conceptually generated and which is usually carried out by a human; and the decision stage (convergent stage) where the solutions are evaluated and selected, and again, the decision is made by a human.

Furthermore, computational systems that try to mimic the functionality of the design process must face the difficulty of quantifying the criteria for evaluating in the problem domain the solutions obtained (security, ease of use acceptance by clients, or any other) and their relative importance in case of conflicts. Out of them, the most complicated criterion to take into account is the aesthetic appropriateness of a design as it is a function of the observer's subjectivity. It usually leads to many possible evaluations, which are often very difficult to translate into criteria in the implementation domain.

In this line several authors have addressed the problem of aesthetically evaluating morphologies with the aim of automating their design process. They have done so in an interactive manner by generating different genetic algorithm based applications. Some of them go all the way back to Dawkins and his Biomorphs where users decided in an interactive way what drawings represented a real insect in two dimensions [1]. Later on, in his coffee tables application [2], Peter Bentley established the basis for the application of evolutionary techniques to creative and functional design of three dimensional objects.

In addition to these two key references, there are other papers having to do with Evolutionary Interactive Morphological Design, where solutions to particular problems are provided through the application of artistic and personal criteria. The common thread to all of them is their constrained application to certain stages of the design process and thus their lack of versatility. Some examples in this line are:

- Design of sculptures through the subjective participation of the users, developed by Duncan Rowland [3].

- Digital image processing [4]. 
- Musical composition creation [5], [6].

- Automatic figure design [7].

- Generation of artistic designs [8], [9], [10], [11], [12].

- Gesture generation in three dimensional characters for virtual worlds [13].

- Morphology and behavior generation [14], [15].

- Generation of animations [16].

In the work presented here, an aesthetic design module has been integrated in a general automatic design system. The environment allows for the design of systems taking into account their engineering specifications and the opinion of users and/or members of the design team both in the divergent and in the convergent stages of the design process.

\section{AUTOMATIC DESIGN ENVIRONMENT}

The environment for the automatic design of systems and products that has been developed aims at integrating all of the stages of the design process (definition and analysis of the problem, synthesis and analysis of the design and specification of the solution) by means of the application of advanced search and analysis techniques, such as genetic algorithms. This way, the design process is formulated as an optimal solution search process in a high and variable dimensionality solution space.

The integration of this type of techniques in the design environment permits liberating the human designer from its classical function as a solution seeker, reducing its participation to the specification of the problem. Thus, creativity is increased, the manageability of the problems is improved, more varied and higher quality solutions are possible (as these algorithms work directly over parameters in the problem domain) and the fictitious constraints induced by the limitations of the designer are minimized. These effects become critically important when the design of complex, non linear or multidimensional structures requiring the interaction of multiple different knowledge sources is addressed.

Regarding the last aspect, it is important to note that the modularity proposed for the environment makes it possible to evaluate the fitness of the different design proposals using two types of parameters: technical, objective or engineering parameters and parameters that are purely subjective. The former are defined by simulation tools integrated in the design environment. These tools can be commercial simulators (mechanical, hydrodynamic, control or other) or computer code developed ad hoc. The latter imply the existence of human-machine interfaces for the evaluation of subjective fitness. These interfaces must allow humans to provide an evaluation of the partial solutions obtained by the system in a natural and transparent manner. The role of evaluator can be carried out by an expert team or a significant set of final users that represent the profile of the public for which the product is being developed.

This capability of handling technical and subjective parameters for evaluating solutions provides the design environment with a certain concurrent character. It can address anything from purely aesthetic evaluations to evaluations that take into account technical norms and specifications.

On the other hand, all of these capabilities imply that the environment requires large computational resources due to its complexity, its application generality for synthesizing designs starting from zero and its automatic character. For this reason it has been designed so as to make its computational distribution on a heterogeneous PC cluster possible.

In addition, its versatility is guaranteed by its modular structure. This aspect provides for its easy adaptation to each particular problem in a reasonable amount of time by means of the integration of the most appropriate evaluation software set for the particular task and the modification of the parameters of the search procedure

Thus the Basic structure of the design system comprises three blocks:

- Solution search block.

- Decision making block (evaluation with or without human participation).

- Computational distribution block.

Figure 1 displays the functional diagram of the Evolutionary Environment for Automatic Design considered in this paper.

The decision Module is made up of a set of simulators and man-machine interfaces (MMIs) that are integrated into a single functional unit with the aid of the coordinator. The outputs of each one of the simulators are inputs to a fitness function that is also a part of the module.

The output of the fitness function is a fitness value or vector used by the evaluation block in the Search Module. The Genetic operators permit obtaining a new solution population with a higher fitness using the fitness of the individuals in the previous one.

Every time a new generation of solutions is obtained, a decoding process is necessary. This process translates the language of the search module (genotype) into that of the decision module (phenotype). 


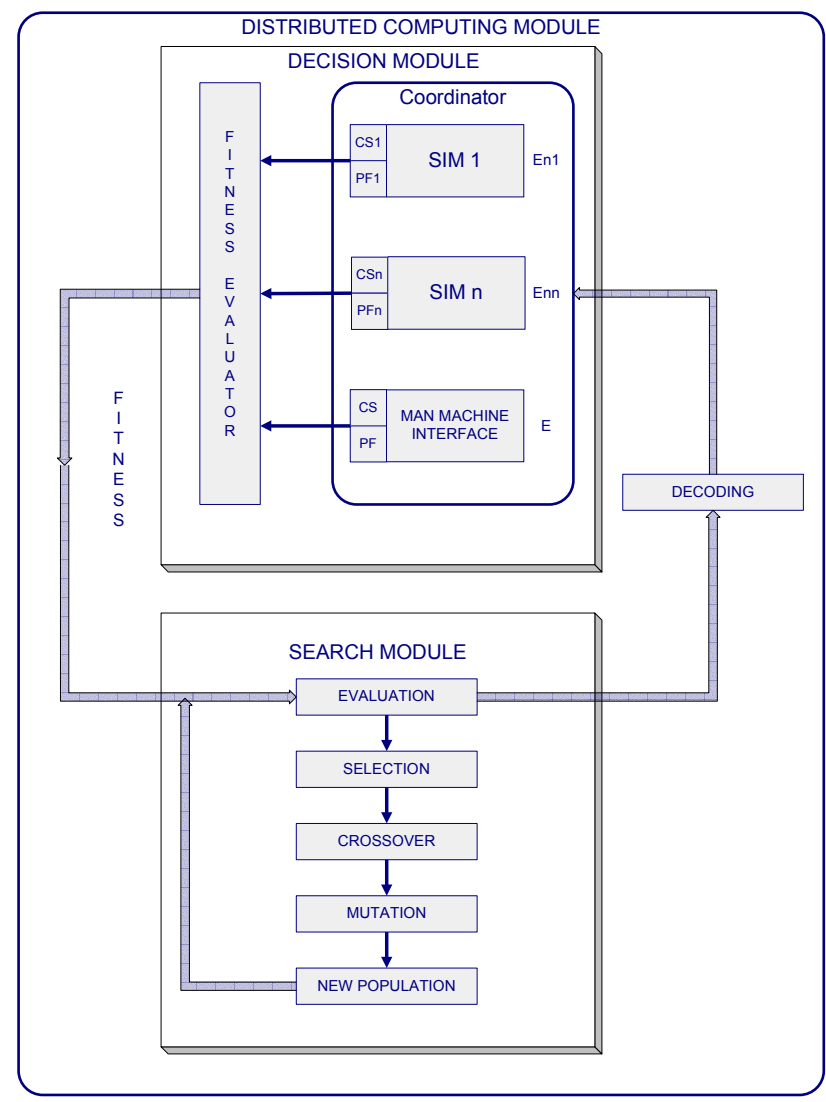

Fig. 1: Evolutionary Automatic Design Environment

The decision module comprises the following elements:

- Simulator Encapsulator $\left(E_{n}\right)$ :

Implements a Standard interface between the Simulator and the environment that includes:

- Decoding of the phenotype to the parameters of the simulation model (weights of a neural network, number and location of machines, connections of electronic systems, surfaces in 3D...).

- Management of simulation parameters (simulation time, number of simulations and others) through scripts or extraction of characteristics from the chromosome.

- Management of orders received by the process coordinator.

- Outputs:

- Commands to other processes or simulators manager by the process coordinator.

- Values of the evaluation of the Simulator that are a part of the fitness function and whose result is handled by the evaluator of the solution search module.

- Parameter Filter $\left(\mathrm{F}_{\mathrm{n}}\right)$ :

Module that selects the parameters that are taken into account in the evaluation function or as control signals for other processes out of all the parameters generated by a Simulator.

\section{- Control Signals $\left(C_{n}\right)$ :}

Signals for the coordination of the collaboration among the different simulation tools.

Summarizing, it can be stated that the combination in the design environment of an advanced exploration strategy, reliable simulators and a man-machine interface for the subjective evaluation of partial solutions and the power of distributed computing implies a radical change in the use of computational tools for assisting in the design process. Thus, we are going from an initial concept of tools for assisting the designer in the design process to a new design system that plays many of the roles traditionally reserved for humans relegating them to a specification function and to subjective evaluation processes.

\section{EXPERIMENTS}

The main objective of this implementation is the integration in the simulation/interaction module of the design environment of a system for the visualization of shapes and a simple interface for the synthesis of three dimensional morphologies through multiple and subjective evaluations by a set of users.

In the example proposed in this paper the shape generation system will create three dimensional shapes defined as instances of parameterized mathematical models. The user will have access to each one of the morphologies generated by the automatic system and will provide an evaluation of its relative appropriateness. The search procedure will then evolve the model depending on the joint fitness obtained through the subjective evaluations provided by the user set. This will lead to a product that should conform to the aesthetic requirements of the group if there is a common set of requirements or to a variety of products when no such common subjective requirements are present.

With regards to the implementation of this strategy, evolutionary algorithms have become an extremely useful tool when addressing problems related with the optimization of products, processes and systems. This is due to their convergence, simplicity and robustness. However, little attention has been paid to them in their application to the interactive design of products. In this implementation we will make use of these algorithms for the search of solutions.

In addition, the three dimensional generation of the shapes is handled by a classical CAD system. This type of systems, even though they have evolved quite a bit, are still computational systems for helping the designer. In this case they will become the base for an automatic design system. Consequently, its classical interface must be inhibited and the interpretation and three 
dimensional representation of the population of morphologies that must be evolved needs to be automated.

In addition to the CAD system we define an interactive interface for presenting the user with the different visual alternatives provided by the CAD system. This interface allows the user to evaluate how much they like or dislike the 3D morphologies generated.

The environment is run on a 51 pc cluster organized as a server and 50 clients. The master coordinates the evolutionary optimization process. Each individual is a three dimensional solid generated automatically by AutoCAD in each client computer and which is genetically encoded by a real valued vector. In the first generation, the server will provide randomly generated chromosomes, which will correspond to random 3D morphologies within the constraints imposed on the CAD system. These morphologies will be constructed and rendered in the clients. The users will evaluate them through a visual interface (in this case it was programmed using MATLAB). This way, in the simulation/interaction module of the design environment we have two collaborating processes, one supported by AutoCAD for the generation of the $3 \mathrm{D}$ shapes and another one for providing the evaluation interface to the user.

The population was made up of 240 individuals and each one of them was encoded as a 20 gene vector (chromosome) defining three dimensional points. The solid (phenotype) was created by passing a surface through all of these points.

The operation of AutoCAD was reconfigured so that instead of being used as a tool by the designer, it would become the virtual implementer of the designs provided as chromosomal values. Thus a set of macros were programmed using VBA so that when each client receives the chromosome arriving from the server, AutoCAD is activated, it processes the data, translating the chromosome into parameters it can use within its drawing functions. It creates the 3D shape and provides a view of it as a jpeg image which is sent to the user interface. Whenever a client finishes processing the three dimensional information of eight individuals, an interface with these eight images presented as two screens of four are provided to the user who is allowed to evaluate the individuals in a relative manner. The user does this by providing values from 0 by 10 for each one of the individual images according to its tastes.

Figure 2 displays the implementation of the Automatic Design Environment for this particular application where de decision module contains the Image constructor and the interface handler.

An image of the screens generated by the user interface is presented in Fig. 3. As commented before, each interface presents 8 morphologies in two groups of 4 and the user provides a relative fitness value for each one of them through a combo box.

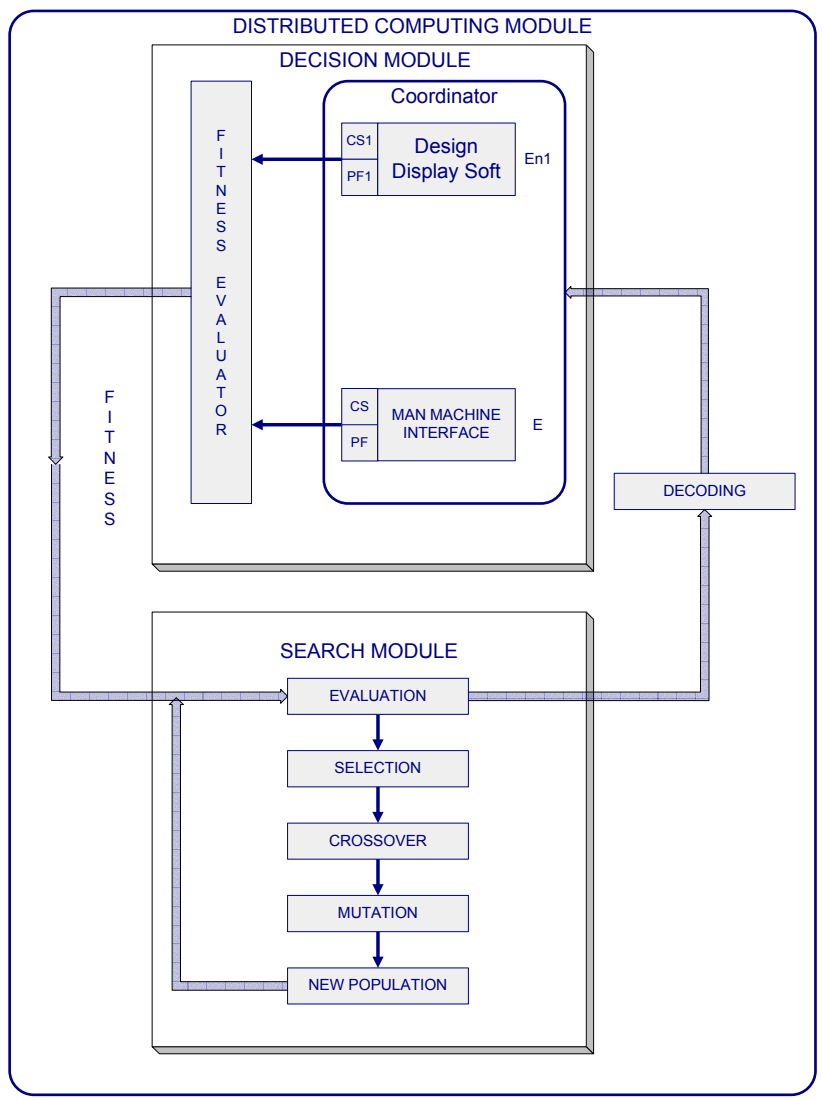

Fig. 2: Diagram of the particular implementation of the Automatic Design Environment for this aesthetic evaluation application

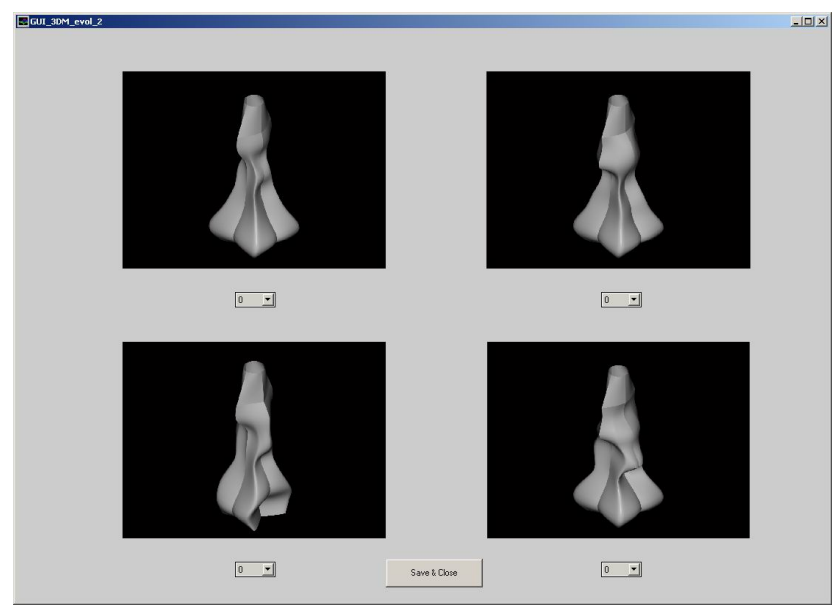

Fig. 3: User Interface

Once the server receives a message indicating the users have evaluated all of the individuals that constitute a population, it compiles the fitness values provided for each individual and through the execution of the corresponding genetic operators (selection, crossover, mutation and population substitution) creates a new population. The mutation probability used in this particular case was $10 \%$, 
crossover takes place $100 \%$ of the time and selection is by tournament selection with a window of 2 to reduce evolutionary pressure. Mutation is taken as the random modification of any of the genes that make up the chromosome. When the fitness of the best individual of the population is similar to the average of the fitness of the population we assume the process has converged.

We carried out two types of tests. In the first experiments we allowed the students to freely choose what they liked better. In the second ones we indicated that we needed mechanical stability and that pyramid like structures would be better. In what follows we describe these two experiments.

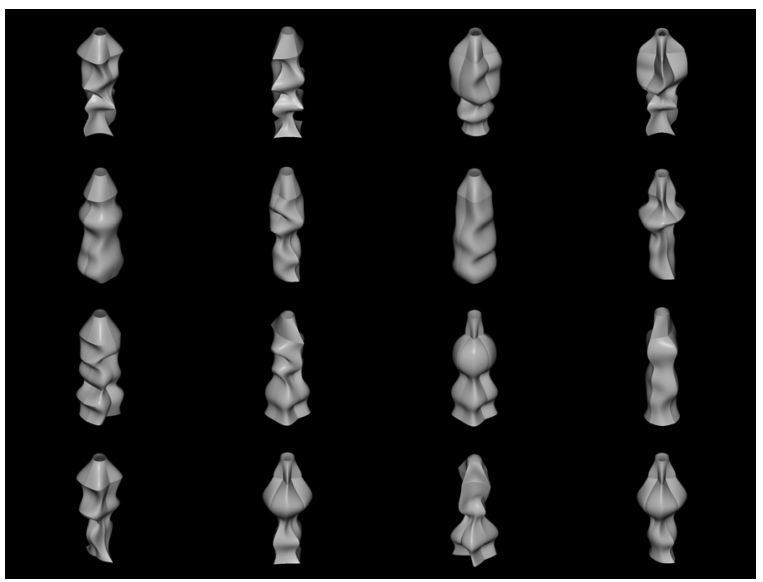

Fig. 5: Case 1, sample of individuals in the last generation

\section{- $\quad$ Case 1}

In this case we tested the system with a group of 10 users without providing any prior evaluation criteria. We let each user choose whatever they liked best. As evolution progressed, and given the fact that the set of users was heterogeneous, with different

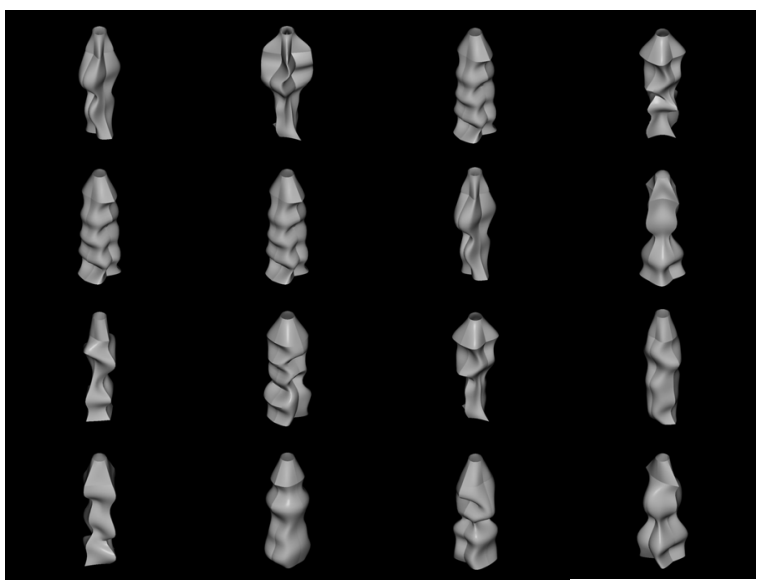

Fig. 4: Case 1, sample of individuals in the first generation

aesthetic sensitivities, the evolutionary process provided a broad spectrum of shapes. More than converging to a common ideal of bottle it just excluded those that were not of the liking of any of the users.

Figures 4 and 5 display the initial random set of shapes and those after 15 generations of evolution.

The results show that there are many aesthetic tastes within the user population with a tendency towards bulbous tops but no consensus on the rest.

As a continuation of the experiment we wanted to see what the system would obtain when there was a certain common ground on aesthetics and that is what we did on the next case.

\section{- $\quad$ Case 2}

For this case a general criterion was provided to the users in terms of having mechanically stable bottles, that is, bottles that would not topple over. In this case, after 15 generations of evolution (figure 7) it was observed that the bottle population became stable and that most of them were pyramidal with slight modifications on the top part.

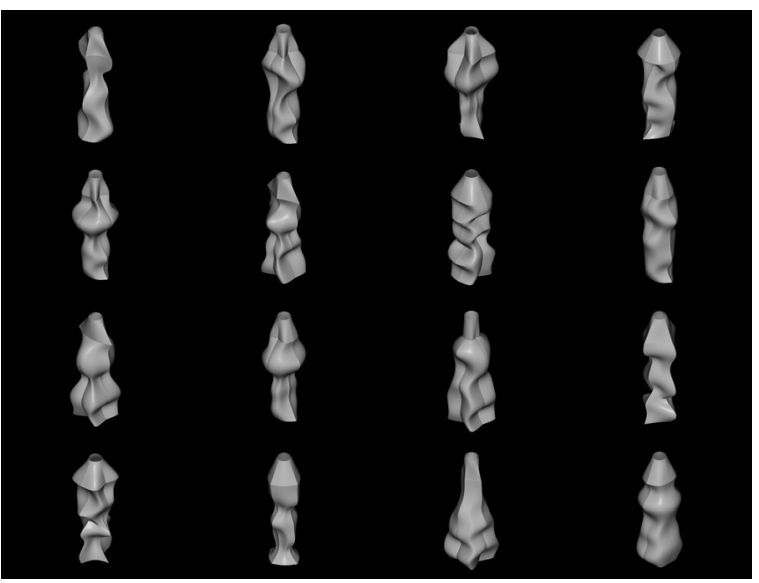

Fig. 6: Case 2, sample of individuals in the first generation

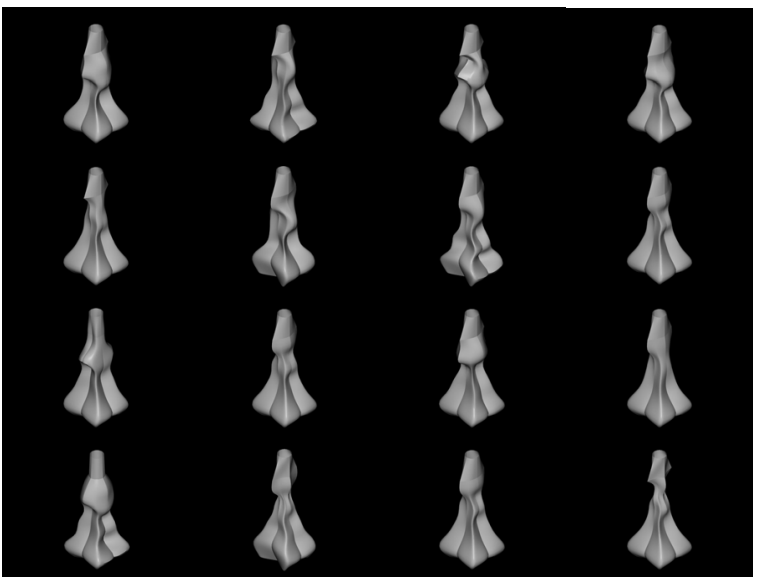

Fig. 7: Case 2, sample of individuals in the last generation

Thus, these results show how common subjective elements can be extracted from a group of users through their interaction with the system during the design process. In fact, if a strong similarity of tastes 
is present in the evaluating users, we see a convergence of the algorithm to basically the same morphology (see Fig. 8).

When this consensus is not present, we see the system extracting some common elements that are used in different combinations. Consequently, the system is capable of automatically extracting the preferences of sets of potential users and combining these in order to obtain designs that please them.

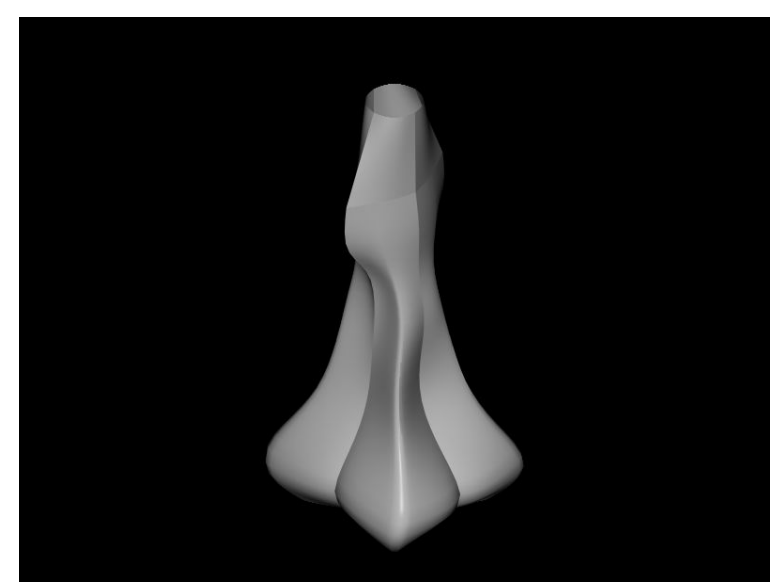

Fig. 8: Individual in the last generation

If this type of subjective evaluations is combined with evaluations obtained from the technical merit of the solutions through simulators in the decision module (as done in [17]) it is possible to generate designs that present the highest potential acceptability by the users within the technical constraints imposed on them. If these evaluations are carried out by experts in the different technical disciplines necessary for the project (engineers, manufacturing, packaging, sales, etc.) who provide their opinion on each of the partial solutions obtained, we have a concurrent design environment.

\section{CONCLUSIONS}

This paper presents a methodology that makes it possible to automatically design products and systems that imply subjective criteria. In this case it was shown that it is possible to interactively design three dimensional morphologies by means of the definition of an automatic interactive design environment that allows human evaluation of shapes generated by a viewing system.

The results show that the idea is very promising as one of the most difficult problems during the design of many types of products is deciding what aesthetic criteria are accepted by the final user and which will produce a competitive edge in a given segment of the market. The tool proposed here provides an indication of how the concurrent design of products can be carried out combining subjective and technical criteria without having to use a human designer to filter them into a final design.
This new concept makes the environment a system for assisting in the design as opposed to classic CAD systems which can be termed systems for assisting the designer.

\section{ACKNOWLEDGEMENTS}

This work was funded by the MCYT of Spain through project VEM2003-20088-C04-01 and Xunta de Galicia through project PGIDIT03TIC16601PR.

\section{REFERENCES}

[1]. Dawkins, R., The Blind Watchmaker, Longman Scientific \& Technical Pub, 1986.

[2]. Bentley P.J., From Coffee Tables to Hospitals: Generic Evolutionary Design, Evolutionary design by computers, Morgan-Kauffman, pp. 405-423, 1999.

[3]. Rowland, D., Biocca, F., Evolutionary cooperative design between human and computer: implementation of the genetic sculpture park, Proceedings of the fifth symposium on Virtual reality modeling language (Web3D-VRML), 2000.

[4]. Ngo, J.T., Marks J., Spacetime Constraints Revisited. Computer Graphics, Annual Conference Series, pp. 335-342, 1993.

[5]. Moore, J.H., GAMusic: Genetic algorithm to evolve musical melodies, http://wwwcgi.cs.cmu.edu/afs/cs/project/airepository/ai/area s/genetic/ga/systems/gamusic/0.html, 1994.

[6]. De la Puente, A. O., Alfonso R. S., Moreno M. A, Automatic composition of music by means of grammatical evolution, Proceedings of the 2002 conference on APL: array processing languages: lore, problems, and applications, New York: ACM Press, 2002.

[7]. Vico F.J., Veredas F.J., Bravo J.M., Almaraz J., Automatic design synthesis with artificial intelligence techniques. Artificial Intelligence in Engineering 13, pp. 251-256, 1999.

[8]. Santos, A., Dorado, J., Romero, J., Arcay, B., Rodríguez, J., Artistic Evolutionary Computer Systems, Proceeedings of the Genetic ad Evolutionary Computation Conference Workshop, Las Vegas, 2000.

[9]. Unemi, T., SBART 2.4: an IEC Tool for Creating 2D images, movies and collage, Proceedings of the Genetic and Evolutionary Computation Conference Program, Las Vegas, 2000.

[10]. Whitelaw M., Breeding aesthetic objects: art and artificial evolution, Creative evolutionary systems, Morgan Kaufmann Publishers Inc., San Francisco, CA, 2001

[11]. Todd S., Latham W., Evolutionary Art and Computers, Academic Press, Inc., Orlando, FL, 
1994

[12]. Thomas D., Aesthetic selection of developmental art forms, Proceedings of the eighth international conference on Artificial life, p.157-163, December 09-13, 2002

[13]. Berlanga, A., Isasi, P. Segovi,a J., Interactive Evolutionary Computation with Small Population to Generate Gestures in Avatars, Proceedings of GECCO 2001, Artificial Life, Adaptative Behavior and agents, 2000.

[14]. Sims K., Evolving 3D Morphology and Behaviour by Competition, Artificial Life IV proceeding, MIT Press, 1994.

[15]. Sims, K., Evolving 3D Morphology and Behaviour Schemes, Proceedings of the 5th Annual Conference on Evolutionary Programming, Cambridge, MA: MIT Press, pp. 121-129, 1994

[16]. Sarafopoulos A., Buxton B.F., Evolutionary algorithms in modeling and animation, Handbook of computer animation, SpringerVerlag, London, 2003

[17]. Lamas A., Fernández Ibarz J., López Peña F. \& Duro R.J. Evolutionary Design of a MultiANN Control Structure for a Submersible Catamarans, CIMSA 2003 IEEE International Symposium on Computational Intelligence for Measurement Systems and Applications. V-1, pp. 32-37, July, 2003.

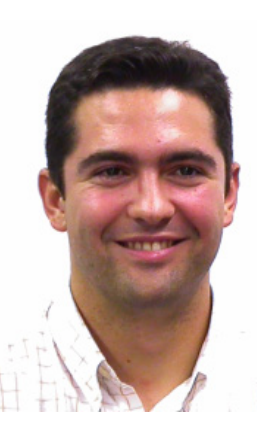

Adolfo Lamas Rodríguez received a M.S. degree in Industrial Engineering from the University of Vigo, Spain in 1999, and a PhD in Industrial Engineering from the University of A Coruña in 2004. He is a member of the Integrated Group for Engineering at the University of Coruña, Spain. His research interests include process automation and evolutionary design.

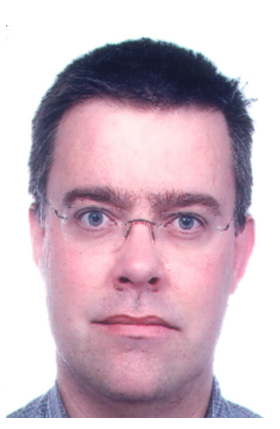

Richard J. Duro received the B.S. and M.S. degree in Physics from the University of Santiago de Compostela, Spain, in 1989, and a PhD in Physics from the same University in 1992. He is currently a Profesor Titular in the Department of Computer Science and member of the Integrated Group for

Engineering at the University of A Coruña, Spain. His research interests include higher order neural network structures, signal processing and autonomous and evolutionary robotics. $\mathrm{He}$ is a Senior member of the IEEE. 